\title{
Cereal signs analysis associated with color on digital images
}

Komyshev E.G. ${ }^{*}$, Genaev M.A. ${ }^{1}$, Smirnov N.V. ${ }^{2}$, Afonnikov D.A. ${ }^{1,2}$

${ }^{1}$ Institute of Cytology and Genetics, SB RAS, Novosibirsk, Russia

${ }^{2}$ Novosibirsk State University, Novosibirsk, Russia

*e-mail:komyshev@bionet.nsc.ru

The color and textural characteristics of the ear and grains are associated with a number of phenotypic traits that are important for selection. The color of the ear can be associated with the stage of plant development, the content of various metabolites in the plant tissues, or the stressful effects of the environment. Textural signs can be used to detect pathogens or anatomical parts of the spikelet. We propose the results of an evaluation of the method for classifying cereal grains based on color and textural characteristics using machine learning approaches. As part of this work, a data set was created in which images of grains of different genotypes are presented: parental forms of the ITMI mapping population that carry nonallelic genes of red grain; the Opata- 85 sort carries the R3 gene (chromosome 3BL), and the synthetics R-93 carries the R1 gene (3DL chromosome). Among 110 recombinant lines, there are genotypes - carriers of one or two genes of the color, or white grain (recessive alleles of both genes), on which the nature and intensity of the color depends. We calibrated the color of images by method used in epilluminous microscopy using the target ColorChecker Mini Classic, which was placed in the area of each frame. Such a correction made it possible to eliminate color distortions in the image arising due to different lighting conditions. The grains in the image were recognized by the method proposed by us earlier and implemented in the SeedCounter mobile application. As a result, a set of metrics that are suitable for describing the texture and color of wheat grains was determined. We evaluated the importance of features in the task of classifying grains according to color characteristics and implemented the calculation of these features in the SeedCounter mobile application. Acknowledgements: This work was supported by the Russian Science Foundation, the project 17-74-10148. Authors are grateful to Pshenichnikova T.A. for plant seed material. 